\title{
A VIVÊNCIA DO CUIDADO MATERNO A UMA LACTENTE COM EPIDERMÓLISE BOLHOSA
}

\section{EXPERIENCE OF MATERNAL CARE TO AN INFANT WITH EPIDERMOLYSIS BULLOSA}

\section{EXPERIENCIA DEL CUIDADO MATERNO DE UN LACTANTE CON EPIDERMÓLISIS BULLY}

Ronaldo Antonio da Silva ${ }^{1}$, Renata Emily da Silva dos Santos ${ }^{2}$, Lidiane Cristina da Silva Alencastro ${ }^{3}$, Karina Nonato Mocheuti ${ }^{4}$, Thalison Fernandes Pinheiro ${ }^{5}$, Fabiane Blanco Silva Bernardino ${ }^{6}$

\section{RESUMO}

Objetivo: Compreender o cuidado materno realizado a uma lactente com Epidermólise Bolhosa. Método: Estudo de caso único com abordagem qualitativa realizado com a mãe de uma lactente com seis meses de idade e diagnóstico clínico de Epidermólise Bolhosa. A recolha dos dados ocorreu em dois encontros em domicílio da participante por meio de entrevista em profundidade e observação. Empregou-se a análise de conteúdo do tipo temática. Resultados: A partir das vivências do cuidado materno empreendido a lactente, emergiram duas categorias temáticas: dificuldades enfrentadas no cuidado materno à lactente com Epidermólise Bolhosa e aspectos que fortalecem o cuidado materno à lactente com Epidermólise Bolhosa. Considerações finais: Os profissionais de saúde precisam proporcionar suporte e esclarecimentos à mãe e à família diante das dificuldades que porventura surgirem, bem como incentivar, valorizar e explorar os aspectos que fortalecem o cuidado materno nesse contexto de adoecimento crônico e raro.

Descritores: Doenças Raras; Epidermólise Bolhosa; Lactente; Cuidadores; Enfermagem.

\section{ABSTRACT}

Objective: To understand the maternal care provided to an infant with Epidermolysis Bullosa. Methodology: This is a single case study with a qualitative approach carried out with the mother of a six-month-old infant diagnosed with Epidermolysis bullosa. Data collection took place in two meetings at the participant's home through an in-depth interview and observation. Thematic and content analysis was used. Results: From the experiences of maternal care given to the infant, two thematic categories emerged: difficulties faced in maternal care for infants with Epidermolysis Bullosa and aspects that strengthen maternal care for infants with Epidermolysis Bullosa. Final considerations: Health professionals need to provide support and clarification to the mother and family in view of the difficulties that may arise, as well as encourage, value and stimulate the aspects that strengthen maternal care in this context of a chronic and rare disease.

Descriptors: Rare Diseases; Epidermolysis Bullosa; Infant; Caregivers; Nursing.

\section{RESUMEN}

Objetivo: Comprender el cuidado materno que se brinda a un lactante con Epidermólisis Bullosa. Metodología: Estudio de un caso único con abordaje cualitativo realizado con la madre de un niño de seis meses y diagnóstico clínico de Epidermólisis Bullosa. La recolección de datos se llevó a cabo en dos reuniones en el domicilio del participante a través de una entrevista en profundidad y observación. Se utilizó análisis de contenido temático. Resultados: De las experiencias de la atención materna realizadas al lactante surgieron dos categorías temáticas: las dificultades enfrentadas en la atención materna del lactante con Epidermólisis Bullosa y los aspectos que fortalecen la atención materna del lactante con Epidermólisis Bullosa. Consideraciones finales: Los profesionales de la salud deben brindar apoyo y aclaraciones a la madre y a la familia ante las dificultades que puedan haber surgido, así como incentivar, valorar y fortalecer los aspectos que fortalecen la atención materna en este contexto de enfermedades crónicas y raras. Descriptores: Enfermedades Raras; Epidermólisis Ampollosa; Lactante; Cuidadores; Enfermería.

${ }^{1}$ Enfermeiro. Mestre em Enfermagem. Universidade do Estado de Mato Grosso. ${ }^{2}$ Graduanda em enfermagem. Universidade do Estado de Mato Grosso. ${ }^{3}$ Enfermeira. Doutora em Ciências da Saúde. Universidade Federal de Mato Grosso. ${ }^{4}$ Enfermeira. Mestra em Educação. Universidade do Estado de Mato Grosso. ${ }^{5}$ Enfermeiro. Mestre em Enfermagem. Secretaria Municipal de Saúde de Nova Olímpia. ${ }^{6}$ Enfermeira. Doutora em Ciências da Saúde. Universidade Federal de Mato Grosso.

Como citar este artigo:

Silva RA, Santos RES, Alencastro LCS, et al. A Vivência Do Cuidado Materno A Uma Lactente Com Epidermólise Bolhosa. Revista de Enfermagem do Centro-Oeste Mineiro. 2020;10:e4133. [Access___ Available in:__. DOI: http://doi.org/10.19175/recom.v10i0.4133 


\section{INTRODUÇÃO}

As doenças raras geralmente são desencadeadas por mutações genéticas que levam a alterações fisiopatológicas no organismo e caracterizam-se por apresentar baixa prevalência ${ }^{(1)}$. Atualmente são conhecidas de seis a oito mil doenças raras, $6 \%$ a $10 \%$ do total das doenças mundiais ${ }^{(1)}$. Dentre tais doenças, pode-se destacar a Epidermólise Bolhosa (EB) do tipo hereditária, uma genodermatose não contagiosa, sistêmica, com grande variação clínica que tem a fragilidade epitelial como principal característica ${ }^{(2-}$ 4).

A evolução clínica da EB depende do tipo e subtipo, cujas formas graves podem atingir vários órgãos e apresenta alta probabilidade de óbitos nos primeiros anos de vida ${ }^{(2,4)}$. As complicações variam de acordo com a severidade da doença e incluem alterações, em quase todos os sistemas do organismo, com maior predisposição para o desenvolvimento de neoplasias dermatológicas, retardo no crescimento, desnutrição e inflamação crônica ${ }^{(2,4-5)}$.

Em relação aos dados epidemiológicos da EB, podem variar de acordo com o país e ser de difícil precisão, em virtude da falta de um sistema de informação específico para avaliar a sua distribuição(6). No Brasil, não há registros epidemiológicos, pois a doença não consta na lista de notificação compulsória e agravos do Ministério da Saúde, o que impede conhecer sua distribuição. Em contrapartida, nos Estados Unidos, verifica-se que a prevalência é de 11,07 casos por um milhão de habitantes, e a incidência de 19,57 casos por um milhão de nascidos vivos ${ }^{(6)}$.

Em decorrência da complexidade e cronicidade da EB, a continuidade do cuidado da criança, após a alta hospitalar, é atribuída prioritariamente à família. Nesse contexto, a mãe se destaca como a principal cuidadora dos filhos com diagnóstico de EB, assumindo esse papel complexo e contínuo na rotina familiar ${ }^{(7-10)}$. Ademais, os resultados de uma revisão recente sobre o cuidado familiar à criança e ao adolescente com EB evidenciou que a mãe, em especial, constrói conhecimentos e habilidades necessários, extraindo-os de sua própria experiência cotidiana, considerando as particularidades impostas pela condição de adoecimento do filho ${ }^{(9)}$.

Nesse contexto, questiona-se: como a mãe vivencia o cuidado da filha lactente diagnosticada com EB? $O$ desenvolvimento do presente estudo torna-se relevante pela aproximação que faz com a vivência da maternidade, seja quanto aos aspectos difíceis ou quanto aos favoráveis presentes no nascimento e cuidado de uma criança com o diagnóstico de EB. Tal saber poderá auxiliar os profissionais da enfermagem para o cuidado centrado na família. Assim, dada à complexidade de vivenciar a maternidade de uma criança diagnosticada com doença rara e os cuidados específicos que ela demanda, notadamente no primeiro ano de vida, este estudo tem como objetivo compreender o cuidado materno realizado a uma lactente com EB.

\section{MÉTODO}

Considerando a raridade da EB e, consequentemente, a representatividade do caso investigado, optou-se pelo método de estudo de caso único com abordagem qualitativa. Assim, o presente estudo tem, como marco teórico e metodológico, os pressupostos do estudo de caso, levando em consideração conceitos próprios de uma investigação de um fenômeno em seu contexto real|(11).

O caso focalizado nessa investigação foi identificado, por meio das mídias sociais, e o processo de seleção estabeleceu os seguintes critérios de inclusão: ter a idade igual ou superior a dezoito anos; ser mãe de uma criança menor de um ano de idade com diagnóstico clínico ou genético de $E B$, por ser considerado o período mais crítico para o cuidado; ser usuária do Sistema Único de Saúde (SUS); e residir no estado de atuação dos pesquisadores, com o intuito de viabilizar a coleta de dados.

Para a recolha dos dados empíricos, foram utilizadas as técnicas de Entrevista em Profundidade (EP) e Observação. A EP é entendida como uma conversa com intencionalidade, configurando-se como uma estratégia de entrevista flexível, sem a necessidade de roteiros e que busca memórias de forma não linear, respeitando a dinamicidade da narrativa do sujeito ${ }^{(12)}$. Assim, a questão norteadora 'Como tem sido para você vivenciar o cuidado da sua filha com EB?' foi utilizada para permitir o aprofundamento paulatino nas memórias e narrativas da participante do estudo ${ }^{(12)}$.

O trabalho de campo transcorreu por encontros em domicílio da participante com datas e horários previamente agendados, pautados pela sua preferência e pela menor interferência no cotidiano de vida, em respeito à sua privacidade. Portanto foram marcados dois encontros, entre fevereiro e março de 2020. Logo após cada um 
deles, no sentido de complementar e enriquecer a $E P$, efetuaram-se os registros de observação, com a percepção individual dos pensamentos, intuições dos pesquisadores, bem como as manifestações da participante, tais como olhares, gestos, posturas, silêncios, além da descrição dos objetos e cenários ${ }^{(12)}$.

Para a organização dos dados, todo o material empírico coletado foi digitado no Diário de Pesquisa ${ }^{(13)}$, que contém as transcrições das EP com as narrativas na íntegra e os registros de observação. O corpus de análise deste estudo constituiu em um arquivo, digitado no Word, com 56 laudas. Empregou-se a análise de conteúdo do tipo temática, seguida pela organização da análise, codificação, categorização e inferência ${ }^{(14)}$, procedimento que fez emergir duas categorias temáticas: dificuldades enfrentadas no cuidado materno à lactente com EB e aspectos que fortaleceram o cuidado materno à lactente com EB.

Este estudo foi apreciado e aprovado pelo Comitê de Ética em Pesquisa da Universidade do Estado de Mato Grosso (CEP/UNEMAT) sob o CAAE: 26311819.2.0000.5166 e parecer número 3.779.764. O compromisso ético foi intermediado pela assinatura da participante e do pesquisador coordenador do Termo de Consentimento Livre e Esclarecido, respeitando os preceitos éticos de pesquisas com seres humanos estabelecidos na resolução no 466/2012 do Conselho Nacional de Saúde do Brasil.

\section{RESULTADOS}

O caso em estudo teve como centro de observação e análise a mãe de uma lactente do sexo feminino com seis meses de idade e diagnóstico clínico de EB. A participante é uma mulher com 26 anos, de cor parda, religião protestante, estudante universitária, casada e residente com o pai biológico de sua única filha, em um município do interior do estado de Mato Grosso-MT, Brasil. De acordo com as narrativas da mãe, a lactente nasceu de parto cirúrgico, com aplasia cutânea no membro inferior direito, em um hospital do interior do estado e foi transferida, para o hospital de referência na capital, após sete dias de internação.

Em seguida ao nascimento e diagnóstico da filha, a mãe relatou que o apoio, principalmente de seus pais e do esposo, foi fundamental ao cuidado da lactente. Após a alta hospitalar, a mãe rememora que só conseguiu assumir de forma efetiva no domicílio todos os cuidados requeridos pela filha depois de transcorrido um mês. Tendo por base as vivências dessa mãe, a análise do material empírico deu origem à construção de duas categorias temáticas, as quais contribuíram com a discussão sobre os aspectos que dificultaram e os que fortaleceram a aquisição dos conhecimentos e habilidades necessários para o cuidado materno à lactente com EB.

\section{Dificuldades enfrentadas no cuidado materno à lactente com Epidermólise Bolhosa}

Os primeiros impasses, relatados pela mãe no cuidado de sua filha com EB, iniciaram-se logo após o parto. $O$ desconhecimento sobre a doença rara pelos profissionais de saúde atuantes no hospital de nascimento da lactente se apresentou como a primeira dificuldade a ser enfrentada. A mãe frisa que a suspeita do diagnóstico clínico de EB foi inferido entre dois ou três dias após o parto, quando um profissional médico buscou esclarecimentos com outros profissionais do hospital de referência da capital do estado, conforme apresenta à narrativa: "Os médicos também não sabiam o que estava acontecendo. Ninguém sabia, só achavam que ela estava com alguma infecção, mas dava negativo! Essa médica fez uma pesquisa com outros médicos do [hospital da capital] e eles chegaram [em] uma possível conclusão que ela poderia ter a Epidermólise Bolhosa (Mãe)".

Segundo a participante do estudo, mesmo tendo sido realizado o diagnóstico clínico, a equipe de enfermagem desconhecia as especificidades necessárias para o cuidado de uma criança com EB. A mãe rememora que "As enfermeiras que estavam no momento cuidando da gente, elas não entendiam também a gravidade da doença [...] queriam tratar ela como qualquer outro paciente, mas infelizmente ela não era outro paciente, ela tinha EB (Mãe)".

Passado o período de internação hospitalar, as dificuldades mencionadas pela participante do estudo quanto ao cuidado domiciliar apresentaram relação direta com as particularidades do contato/toque com a pele da lactente com EB: "As feridas apareciam como se fosse mágica. Ela estava bem, a gente olhava para a pele dela estava lisinha, olhava cinco minutos depois já tinha uma bolha ou uma ferida [...] 0 jeito que pegava nela ia dando os traumas, então a gente achava que era uma infecção muito, muito grave que estava descamando a pele dela (Mãe)".

Essa especificidade também levou a mãe a ter dificuldades na amamentação, que foi 
interrompida no terceiro dia após o nascimento, pela rápida evolução clínica da doença: "Começamos tentar a amamentação. Parecia que ela estava com fome! Aí toda vez que a gente tentava ela chorava, chorava e não queria. Mais quando ela não mamava, ela também chorava. [...] Eu comecei a perceber que ela estava com dor, ela queria, mas ela estava com dor. Daí então que eu percebi que ela estava com ferida na boca. [...]. A insistência no momento lá feriu a sua boca, deu a lesão e criou a bolha de sangue (Mãe)".

Ainda, no que se refere aos cuidados maternos, a complexidade do curativo também foi citada como um aspecto que dificultou o cuidado materno e, consequentemente, a construção das habilidades para lidar com a doença. A mãe recorda o momento em que foi dada à alta hospitalar e não conseguia fazer os curativos no pé da filha, local que nasceu com extensa aplasia cutânea. O procedimento era demorado, causava muita dor na lactente, e presenciar isso causava angústia e sofrimento para a mãe: "[A lactente] teve alta e daí foi uma verdadeira guerra aprender a cuidar dela. Aprender a fazer os curativos. E no momento eu não conseguia fazer os curativos. No primeiro mês eu não entrava no quarto. Quando ia fazer o curativo no pé dela eu já saía. Ela chorava muito. Tentava não escutar, porque era desespero não poder fazer nada, não poder tirar a dor. Porque nada adiantava naquele momento (Mãe)". "[...] Ela chorava muito e, é assim, cerca de três a quatro horas pra a fazer os curativos. Ela tinha muita dor, às vezes, fazia cocô de dor (Mãe)".

Além disso, as narrativas da mãe apontam para a sua necessidade de ser ouvida pelos profissionais de saúde. A falta de acolhimento, caracterizada pela participante como a não escuta de suas necessidades, inquietações e apreensões, foi indicada como outro aspecto que dificultou o cuidado de sua filha com EB. Na vivência apresentada neste estudo, a interação estabelecida pela mãe com os profissionais de saúde, portanto careceu de escuta e diálogo: "Tem que escutar o que a mãe fala ali naquele momento. Porque tem muitas pessoas, enfermeiros e médicos que não escutam. Eles acham que os estudos deles [é] o que importa. Às vezes, a gente reclama de alguma coisa não é frescura. Tem muitos profissionais que acham que a gente está ensinando, mas não é isso. A gente quer o melhor pra nossa filha. Falo para vocês [profissionais de saúde] só ouvirem mais o paciente! (Mãe)".
A despeito da aquisição de conhecimentos e habilidades, para o cuidado materno na EB, foram identificados aspectos dificultadores, que precisam ser considerados e minimizados pelos profissionais de saúde que prestam assistência à mãe e família. De acordo com o exposto nas narrativas, torna-se essencial que tais profissionais auxiliem no cuidado materno de crianças com EB, dando o suporte adequado a partir da escuta das necessidades das mães e familiares e do que dizem sobre as especificidades da EB. Assim, segundo a participante do estudo, também houve alguns saberes e condutas que fortaleceram o cuidado materno à lactente.

\section{Aspectos que fortaleceram o cuidado materno à lactente com Epidermólise Bolhosa}

De acordo com a participante do estudo, em sua vivência no cuidado da lactente com EB, também houve fatores que contribuíram para o fortalecimento do cuidado. A esse respeito, o apoio familiar recebido, principalmente pela mãe, foi um importante suporte desde o nascimento da filha e apresentou-se como o primeiro elemento potencializador do cuidado.

A mãe narra que, no primeiro mês de vida da lactente, não foi capaz de realizar os curativos na filha e, nesse período de enfrentamento da doença, a família assumiu a responsabilidade de fazê-los diariamente. Desse modo, o apoio familiar recebido, logo após o nascimento da lactente, favoreceu a construção das habilidades técnicas para cuidar e, com o passar do tempo, a mãe foi capaz de assumir seu papel no cuidado de sua filha: "Graças a Deus tive minha mãe e meu padrasto. Eles foram bem mais fortes que eu. Porque [no] primeiro mês eu não entrava no quarto eu não conseguia ver os machucados dela, não conseguia ver ela. Ela chorava cerca de uma a duas horas sem parar na hora dos curativos (Mãe)". "[O curativo] da mãozinha a gente já fazia, mais do pé mesmo, fui fazer depois de um mês. Já estava um pouco cicatrizado. Não era um machucadinho assim, era uma coisa funda, tinha que lavar que sangrava com frequência (Mãe)".

Somando-se ao apoio familiar nesses momentos de dificuldades, outro elemento que se mostrou potencializador do cuidado materno à lactente com EB veio da aquisição de conhecimentos da mãe pela internet. Em sua vivência, a participante do estudo menciona a busca por informações disponíveis on-line sobre a EB imediatamente após o diagnóstico clínico de sua filha. Segundo as narrativas da mãe, essa 
forma de buscar conhecimento começou ainda durante a internação hospitalar: "Quando a médica falou que era aquela doença eu, como mãe, fui atrás. Pesquisei. Eu lá dentro do hospital, como não dormia, ficava pesquisando e pesquisando. Eu li muito sobre Epidermólise Bolhosa. Eu não queria acreditar que fosse, porque quando você abre a internet é horrível o que você vê (Mãe)".

A partir das narrativas da mãe, foi possível constatar que as pesquisas por informações sobre a EB e as vivências do cuidado diário promoveram a construção de conhecimentos e habilidades exigidos no cuidado de sua filha. Seus relatos evidenciam, inclusive, a sua propriedade e o domínio, ao falar sobre as especificidades da doença experienciada em seu cuidado materno, conforme apresenta a nota de observação: "Explicou tudo, fez questão de falar os preços de cada um e os benefícios de todos os cremes, desde os que eram usados para hidratar, os curativos mais simples até os mais caros. Explicou tudo o que ela sabia sobre a doença. Confesso que fiquei surpresa com todo aquele conhecimento (Notas da observação; 15 de fevereiro, 2020)".

Assumindo o empoderamento sobre a condição da filha, a mãe começou a se tornar referência para outras mães e famílias que enfrentam a mesma situação de adoecimento por EB. Em virtude desse aprendizado, ela passou a ser contatada por intermédio das redes sociais com o objetivo de trocar experiências sobre os cuidados que tinha diariamente com a filha, ofertando apoio às famílias de outras crianças acometidas por essa doença: "Muitas mães entram em contato comigo: como que faz isso, faz aquilo? Igual ontem, uma hora da manhã, eu conversando com uma mãe sobre qual creme era melhor, porque a filha dela estava cheia de ferida (Mãe)".

\section{DISCUSSÃO}

Os resultados do presente estudo assinalaram para as dificuldades e os aspectos que permearam o cuidado materno junto a uma lactente com EB. Desse modo, foi possível compreender que a primeira dificuldade relatada foi imposta, logo após o nascimento de sua filha, ou seja, o desconhecimento por parte dos profissionais de saúde sobre a EB. Pesquisadores ressaltam que o nascimento de uma criança com uma doença rara, como a EB, impõe grandes desafios aos profissionais de saúde por se tratar de uma doença incomum, pouco conhecida e com variação no quadro clínico dos diferentes subtipos $^{(8,15)}$.

O despreparo dos profissionais de saúde, para realizar o diagnóstico clínico e o manejo adequado das lesões na $E B$, também, foi evidenciado em outros estudos, em que, desde o nascimento da criança, os pais vivenciaram sentimentos de ansiedade, insegurança, estresse e solidão, em razão do desconhecimento desses profissionais a respeito da doença ${ }^{(7,9-10)}$. Assim, enfatiza-se a necessidade dos profissionais da saúde estarem amparados por uma literatura específica e atualizados, conforme as diretrizes que orientem as melhores condutas terapêuticas à família, principalmente à mãe, aprimorando conhecimentos e habilidades para a continuidade do cuidado no âmbito domiciliar ${ }^{(8-9)}$. Desse modo, destaca-se a priorização de ações educativas permanentes vindas dos serviços de saúde e divulgação de informações pertinentes para a conscientização sobre EB entre os profissionais da área $^{(7,9)}$.

Outra dificuldade exposta corresponde à dificuldade em realizar o curativo, especificidade própria dos cuidados em EB. Autores ilustram a complexidade do adoecimento por EB, como decorrência da extrema fragilidade dermatológica resultante da evolução clínica da doença ${ }^{(8-9)}$. De acordo com a particularidade da $\mathrm{EB}$, qualquer trauma pode levar ao desenvolvimento de novas bolhas as quais evoluem para feridas, o que exige especificidades no cuidado, principalmente no contato/toque com a pele ${ }^{(8)}$.

Além disso, outro achado importante, mencionado pela participante do estudo como um obstáculo no cuidado materno à sua filha com EB, diz respeito à amamentação, prejudicada pelas lesões de pele típicas da doença. A essa situação autores apontam que pode ser viável introduzir a amamentação, em lactentes com EB, em casos específicos, desde que a mãe seja devidamente orientada e acompanhada por profissionais de saúde capacitados para o cuidado com a EB, processo que deve ser avaliado continuamente $e$ reconsiderado em caso de bolhas e feridas ${ }^{(2-3)}$. 0 caso de um recém-nascido com três dias de nascimento e diagnóstico clínico de EB corrobora com os resultados da pesquisa em tela, pois, em virtude de lesões na gengiva, palato e mucosa labial, houve recusa alimentar por via oral ${ }^{(8)}$.

Outra peculiaridade do cuidado em EB é o curativo, procedimento essencial e diário no tratamento das lesões. Esse procedimento é considerado como a experiência mais sofrida em 
razão da dor, mesmo com a administração de analgésicos e, ao tempo demandado pelos curativos, principalmente nos casos graves ${ }^{(7)}$. Desse modo, em virtude de sua complexidade, os pais encontram dificuldades para a sua execução. Alguns autores observam que, durante o curativo, os pais, em vez de se verem atenuando a dor na criança, eles têm a sensação de causar mais dor e sofrimento a seus filhos ${ }^{(9)}$. Em vista disso, torna-se primordial o suporte e apoio dos profissionais de saúde, durante a hospitalização, por meio do auxílio e oferta de subsídios para a aquisição de conhecimentos e habilidades destinados a garantir a longitudinalidade do cuidado ${ }^{(8)}$.

Ademais, é importante reconhecer a escuta e o diálogo como importantes atributos para a construção do vínculo no atendimento de pessoas e famílias que vivenciam alguma condição de adoecimento crônico e, principalmente, raro. No presente estudo, as narrativas da mãe expuseram, dentre outras dificuldades, a falta de escuta de seus anseios, necessidades e apreensões, por parte dos profissionais de saúde, como um entrave ao cuidado de sua filha com EB. No que se refere especificamente à $E B$, diretrizes reforçam de forma imperativa a necessidade de considerar e envolver a família no cuidado direto da criança ${ }^{(2)}$. No entanto estudo realizado no estado de Mato Grosso, que abarcou a experiência de pais no cuidado do filho com Síndrome de SchinzelGiedion, uma doença rara, também identificou, nas falas dos participantes, a falta de vínculo e diálogo com os profissionais de saúde que atuam de forma pontual, instigando a família a buscar auxílio em outros serviços ou setores para conseguir oferecer o melhor cuidado ao filho(16).

Além dos aspectos que criaram obstáculos ao cuidado materno empreendido à lactente com $E B$, as narrativas da mãe também detalharam aspectos que contribuíram no fortalecimento desse processo, tal como o apoio familiar, referido pela mãe como um fator que auxiliou positivamente na realização do cuidado de sua filha com EB. Autores ${ }^{(15)}$ evidenciam que, apesar de a mãe assumir o papel de principal cuidadora, o apoio familiar recebido no domicílio é fundamental para o enfrentamento nas diferentes situações de adoecimento por condições raras. Além disso, estudo ressalta que a rede social de apoio deve ser construída, ao longo da vida da pessoa que vivencia uma doença rara, pois também se mostra importante para o enfrentamento das adversidades impostas pelo adoecimento, e os profissionais de saúde precisam corroborar nesse processo construtivo ${ }^{(10)}$.

A procura e a disponibilidade de informações na internet sobre a doença também foram descritas pela participante do presente estudo como um fator que auxiliou na aquisição de conhecimento sobre o cuidado a ser realizado a lactente com EB. A busca por esclarecimentos sobre a doença, como a EB, é descrito em outros estudos como algo inerente ao desconhecimento dos profissionais de saúde sobre a condição rara vivenciada pela família(15). Em tal contexto, ao se deparar com uma doença pouco ou completamente desconhecida pelos profissionais de saúde, a mãe, reconhecida como principal cuidadora, precisa buscar informações e construir subsídios para cuidar da criança $a^{(7,9)}$.

Desse modo, assim como no presente estudo, a busca por elucidações em outras fontes, como a internet, bem como as vivências do cuidado diário, proporciona à mãe habilidades $\mathrm{e}$ domínio diante das particularidades da doença ${ }^{(9)}$. No caso em estudo, esse vivenciar da doença também ensejou a construção de uma gama de apoio a outras mães de lactentes com EB, por meio das redes sociais. Autores também destacam que o empoderamento daqueles envolvidos no cuidado de um ente com uma doença rara pode propiciar a troca de experiências com outras famílias na mesma situação, por meio da projeção na realidade do outro, influenciando um ambiente seguro, crucial ao cuidado materno à criança ${ }^{(1)}$.

\section{CONSIDERAÇÕES FINAIS}

$O$ estudo permitiu compreender os aspectos que dificultaram e fortaleceram o cuidado materno de uma lactente diagnosticada com EB. Assim, salientou-se como um dos aspectos dificultadores desse processo o desconhecimento por parte dos profissionais de saúde a respeito da $E B$, as particularidades da doença, a complexidade do curativo e a falta de escuta dos profissionais de saúde na interação com os anseios, necessidades e apreensões da mãe cuidadora em sua experiência com a doença. Como aspectos que fortaleceram a vivência do cuidado materno, destacam-se o apoio familiar, a procura e disponibilidade de informações sobre a doença na internet que, juntamente com as vivências diárias, impulsionaram a aquisição de conhecimentos e habilidades pela mãe para subsidiar o cuidado materno no domicílio, bem como a troca de experiências com outras famílias, por meio das redes sociais. 
Considerando as dificuldades e os aspectos que fortaleceram o cuidado materno compreendidas por meio da vivência da mãe de uma lactente com EB, é crucial reconhecer a necessidade de os profissionais de saúde, especialmente os enfermeiros responsáveis pelo cuidado de enfermagem, atuarem pautando-se na integralidade da assistência, por meio da oferta de suporte e apoio à mãe ante as dificuldades, assim como incentivar e explorar os aspectos que fortaleceram o cuidado nesse contexto de adoecimento crônico e raro.

O presente estudo torna-se relevante por evidenciar as prioridades de mães de lactentes com EB, o que poderá auxiliar os profissionais de saúde e, em específico, os enfermeiros, a construir um cuidado integral tanto na atenção terciária, durante a hospitalização, como na atenção primária, por meio da continuidade do cuidado em domicílio. Ressalta-se, dessa forma, a necessidade de fomentar estudos que investiguem outras doenças raras, no sentido de contribuir e subsidiar a estruturação de políticas e serviços de saúde que contemplem as necessidades das pessoas e famílias que vivenciam este esse tipo de adoecimento.

Como limitações do estudo em tela, destacamos que os achados apresentados correspondem à vivência de apenas uma mãe no cuidado de sua filha com Epidermólise Bolhosa. Em decorrência da raridade da doença e pela ausência de um sistema de informação que rastreie famílias de crianças com doenças raras, assim, identificar esses potenciais participantes ainda é um desafio no Brasil. A esse respeito, estudos futuros podem realizar busca ativa de casos com análises mais aprofundadas por meio da experiência de outras mães com filhos diagnosticados com EB.

\section{REFERÊNCIAS}

1- Luz GS, Silva MRS, DeMontigny F. Doenças raras: Itinerário diagnóstico e terapêutico das famílias de pessoas afetadas. Acta Paul Enferm. 2015;28(5):395-400. DOI: 10.1590/19820194201500067

2- Denyer J, Pillay E, Clapham J. Skin and wound care in epidermolysis bullosa: An expert working group consensus. London: Wounds International; 2017.

3- El Hachem M, Zambruno G, Bourdon-Lanoy E, Ciasulli A, Buisson C, Hadj-Rabia S, et al.
Multicentre consensus recommendations for skin care in inherited epidermolysis bullosa. Orphanet J Rare Dis. 2014;9(1):76. DOI: 10.1186/1750-1172$\underline{9-76}$

4- Laimer M, Prodinger C, Bauer JW. Hereditary epidermolysis bullosa. J Dtsch Dermatol Ges. 2015;13(11):1125-33. DOI: 10.1111/ddg.12774

5- Kim M, Li M, Intong-Wheeler LRA, Tran K, Marucci D, Murrell DF. Epidemiology and outcome of squamous cell carcinoma in epidermolysis bullosa in Australia and New Zealand. Acta Derm Venereol. 2018;98(1):70-6. DOI: 10.2340/00015555-2781

6- Fine J-D. Epidemiology of inherited epidermolysis bullosa based on incidence and prevalence estimates from the national epidermolysis bullosa registry. JAMA Dermatol 2016;152(11):1231.

DOI: 10.1001/jamadermatol.2016.2473

7- Kahraman S, Çiftçi EK, Timuçin A. Determination of caregiving burden of parents providing care to their children with epidermolysis bullosa. Egyptian Journal of Dermatology and Venereology 2017;37(1):1-6. DOI: 10.4103/1110$\underline{6530.207488}$

8- Secco IL, Costa T, Moraes ELL, Freire MHS, Danski MTR, Oliveira DAS, et al. Nursing care of a newborn with epidermolysis bullosa: A case report. Rev Esc Enferm USP 2019,53:e03501. DOI: 10.1590/s1980-220x2018023603501

9- Silva RA, Souza SPS, Bernardino FBS, Alencastro LCS. Family care with children and adolescents with Epidermolysis Bullosa: An integrative literature review. Rev Baiana Enferm. 2020;34:113. DOI: $10.18471 /$ rbe.v34.35781

10- Silva RA, Bernardino FBS, Rocha RPS, Santos RES, Alencastro LCS. A rede de apoio social no cuidado à doença rara e o protagonismo familiar. Res, Soc Dev. 2020;9(10):e1759108385. DOI: $10.33448 /$ rsd-v9i10.8385

11- Yin RK. Estudo de caso: Planejamento e métodos. 5a ed. Porto Alegre: Bookman; 2015.

12- Minayo MCS. O desafio do conhecimento: Pesquisa qualitativa em saúde. 14a ed. São Paulo: Hucitec; 2014.

13- Araújo LFS, Dolina JV, Petean E, Musquim CA, Bellato R, Lucietto GC. Diário de pesquisa e suas potencialidades na pesquisa qualitativa em saúde. Rev Bras Pesqui Saúde 2013;15(3):53-61. DOI: 10.21722/rbps.v15i3.6326

14- Bardin L. Análise de conteúdo. 70a ed. São Paulo: Loyola; 2016.

15- Luz GS, Silva MRS, DeMontigny F. Necessidades prioritárias referidas pelas famílias 
de pessoas com doenças raras. Texto ContextoEnferm. 2016;25(4):1-9. DOI: 10.1590/0104$\underline{07072016000590015}$

16- Soares JL, Araújo LFS, Bellato R. Cuidar na situação de adoecimento raro: Vivência da família e sua busca por amparo dos serviços de saúde. Saúde Soc. 2016;25(4):1017-30. DOI: 10.1590/s0104-12902016162301

Nota: Estudo originado de um Trabalho de Conclusão de Curso apresentado a Faculdade de Ciências da Saúde da Universidade do Estado de Mato Grosso para aquisição do título de bacharel em Enfermagem.

Recebido em: 04/10/2020

Aprovado em: 17/12/2020

Endereço de correspondência: Universidade do Estado de Mato Grosso. Faculdade de Ciências da Saúde. Curso de Bacharel em Enfermagem. Rua Rui Barbosa, no 535, Jardim Eldorado, Diamantino, Mato Grosso, CEP 78400000. 